\title{
Prediction of Temperature Fields induced by Natural Convention in a Cylindrical Enclosure using Fuzzy LS-SVM
}

\author{
Shiyu Zhou ${ }^{1, *}$, Zhengbin $\mathrm{Cao}^{2}$, Guangyue $\mathrm{Du}^{2}$, Xiaoping $\mathrm{Liu}^{2}$ and Yucheng Zhou ${ }^{2}$ \\ ${ }^{1}$ School of Thermal Engineering, Shandong Jianzhu University, Jinan 250101, Shandong, China \\ ${ }^{2}$ School of Information and Electrical Engineering, Shandong Jianzhu University, Jinan 250101, Shandong, China \\ ${ }^{*}$ Corresponding author
}

\begin{abstract}
Support Vector Machines (SVM) is a machine learning algorithm basing on the statistical learning theory. In this study, SVM is used for the prediction of temperature field induced by natural convection in a cylindrical enclosure. Because of the large amount of computing and poor real-time characteristics of conventional SVM, and also to solve the non optimal problem in the whole situation and the over-fitting phenomenon, Fuzzy LS-SVM is adopted. The heat transfer in the enclosure is an unsteady process. The heat transfer process is firstly simulated with CFD software, then part of simulated data is picked for training of LS-SVM model and the rest of data is used for validation of the model. The prediction results are successfully validated from the mean relative error (MRE), max relative error (MAE), mean square error (MSE) and absolute fraction of variance $\left(R^{2}\right)$. Besides, by comparison with artificial neural network based on back propagation (BP-ANN), the fuzzy LS-SVM shows more superior performance in both prediction accuracy and computation efficiency.
\end{abstract}

Keywords—natural convection; support vector machines; fluent; enclosure

\section{INTRODUCTION}

Support Vector Machines (SVM) is a machine learning algorithm basing on the statistical learning theory. Its principle is to construct the optimal separating hyperplane by mapping the input vector into a high-dimensional feature space according to the prior selected non-linear operator [1]. SVM was born in the 90s of the last century [2], since then, it has been applied into more and more widely fields, such as predictions of outdoor/indoor temperature, wind speed, solar radiation, and etc. For example, Paniagua-Tineo, et al [3] presented a novel methodology for daily maximum temperature prediction based on a Support Vector Regression approach. Their study has shown that the SVM algorithm was able to give an accurate prediction of the maximum temperature $24 \mathrm{~h}$ later. Cai, et al [4] established the SVM model and back propagation neural network (BPNN) model of the indoor temperature prediction, respectively. The experimental results indicated that the SVM model performed better than BPNN model in prediction accuracy. Mohandes, et al [5] compared SVM with the multilayer perceptron (MLP) neural networks in performance of predicting wind speed. Results indicated that SVM was superior than the MLP model based on the error analysis between the actual and the predicted data. Chen, et al
[6] studied the feasibility of SVMs in predicting monthly solar radiation with air temperatures based on the statistical learning theory. Comparison showed that the newly developed SVM model performed better than other SVM models and empirical methods.

The more detailed applications of SVM include predictions of thermal and flow fields, entropy production, frost layer thickness and etc. Varol, et al [7] used SVM to estimate the temperature and flow fields in a square porous enclosure heated discretely by three isothermal heaters from the side wall. The results demonstrated that SVM was a useful technique on the estimation of streamlines and isotherms. In their other study [8] forecasting of entropy generation of laminar natural convection in a partially cooled square cross-sectional room was performed with SVM and resulted that the SVM was a strong method for predicting the entropy generation without computational fluid dynamic analysis for all cases. In the study of Zendehboudi, et al [9], four different models including multiple linear regression (MLR), artificial neural network (ANN), least squares support vector machine (LS-SVM), and adaptive neuro fuzzy inference system (ANFIS) were developed to estimate the frost layer thickness on vertical cryogenic surfaces.

The study about natural convective heat transfer in an enclosure has been conducted for several decades because of the classic properties of the problem [10,11]. Das, et al. [12] published a review about this study, they summarized the previous studies on natural convection heat transfer in enclosures with fluid or porous media, which might be triangular, trapezoidal, parallelogrammic, or have an irregular shape with curved and wavy walls. It could be inferred from the review that the previous dominate research approach was based on Computational Fluid Dynamics (CFD) simulation and little study was based on experimental tests [13].

In this paper, SVM is used to predict the temperature field induced by nature convection in an enclosure. However, there is rare study about this issue using SVM, the closest study to this proposition is reference [7], in which, the heat transfer process is a steady process with constant boundary conditions. However, in this paper, the heat transfer is an unsteady process, in which the boundary conditions are changing with time. Therefore, the variation of the temperature field becomes more 
complicated, improving the difficulty of the simulation and prediction. The unsteady heat transfer process in an enclosure is studied in this paper. The process is firstly simulated with CFD software, and then the simulated data is used for the training and testing of the LS-SVM model. Finally the prediction ability of the LS-SVM is compared with that of artificial neural network based on back propagation (BP-ANN).

\section{DESCRIPTION OF THE STUDY}

\section{A. Physical Description}

The studied object in the paper is a cylindrical enclosure whose structure is shown in Figure. 1. As shown in the figure, in the bottom middle of the enclosure, there is a square wood block. The parameters of the cylinder and wood block are shown in Table. 1.

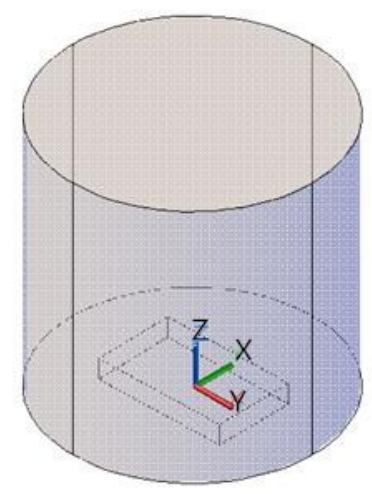

FIGURE I. SCHEMATIC DIAGRAM OF THE CYLINDRICAL ENCLOSURE

TABLE I. PARAMETERS OF THE STUDIED OBJECT

\begin{tabular}{|l|l|l|l|}
\hline & $\begin{array}{l}\text { Cylindrical } \\
\text { wall }\end{array}$ & Block & Fluid \\
\hline Material & Steel & Wood & Air \\
\hline Size $(\mathrm{mm})$ & $\begin{array}{l}\mathrm{H}=200, \\
\mathrm{~d}=2\end{array}$ & $\begin{array}{l}\mathrm{L} / \mathrm{W} / \mathrm{H} \\
100 \times 60 \times 18\end{array}$ & \\
\hline Density $\left(\mathrm{kg} / \mathrm{m}^{3}\right)$ & 8030 & 590 & $\begin{array}{l}\text { Boussinesq } \\
\text { assumption }\end{array}$ \\
\hline $\begin{array}{l}\text { Thermal conductivity } \\
(\mathrm{W} / \mathrm{mK})\end{array}$ & 16.27 & 0.1332 & 0.0259 \\
\hline Thermal capacity $(\mathrm{J} / \mathrm{kgK})$ & 502 & 1633 & 1005 \\
\hline Initial temperature $\left({ }^{\circ} \mathrm{C}\right)$ & 20 & $T_{0}$ & 20 \\
\hline
\end{tabular}

\section{B. Mathematical Description}

The description of the flow and heat transfer in the cylindrical enclosed cavity with conservation equations of mass, momentum and energy are as follows:

$$
\begin{gathered}
\frac{\partial u}{\partial x}+\frac{\partial v}{\partial y}+\frac{\partial w}{\partial z}=0 \\
\frac{\partial(\rho u)}{\partial t}+u \frac{\partial(\rho u)}{\partial x}+v \frac{\partial(\rho u)}{\partial y}+w \frac{\partial(\rho u)}{\partial z}=\frac{\partial}{\partial x}\left(\mu \frac{\partial u}{\partial x}\right)+\frac{\partial}{\partial y}\left(\mu \frac{\partial u}{\partial y}\right)+\frac{\partial}{\partial z}\left(\mu \frac{\partial u}{\partial z}\right)-\frac{\partial P}{\partial x}
\end{gathered}
$$

$$
\begin{gathered}
\frac{\partial(\rho v)}{\partial t}+u \frac{\partial(\rho v)}{\partial x}+v \frac{\partial(\rho v)}{\partial y}+w \frac{\partial(\rho v)}{\partial z}=\frac{\partial}{\partial x}\left(\mu \frac{\partial v}{\partial x}\right)+\frac{\partial}{\partial y}\left(\mu \frac{\partial v}{\partial y}\right)+\frac{\partial}{\partial z}\left(\mu \frac{\partial v}{\partial z}\right)-\frac{\partial P}{\partial y} \\
\frac{\partial(\rho w)}{\partial t}+u \frac{\partial(\rho w)}{\partial x}+v \frac{\partial(\rho w)}{\partial y}+w \frac{\partial(\rho w)}{\partial z}=\frac{\partial}{\partial x}\left(\mu \frac{\partial w}{\partial x}\right)+\frac{\partial}{\partial y}\left(\mu \frac{\partial w}{\partial y}\right)+\frac{\partial}{\partial z}\left(\mu \frac{\partial w}{\partial z}\right)-\frac{\partial P}{\partial z}+\rho g
\end{gathered}
$$

$$
\frac{\partial(\rho T)}{\partial t}+u \frac{\partial(\rho T)}{\partial x}+v \frac{\partial(\rho T)}{\partial y}+w \frac{\partial(\rho T)}{\partial z}=\frac{\lambda}{c}\left(\frac{\partial^{2} T}{\partial x^{2}}+\frac{\partial^{2} T}{\partial y^{2}}+\frac{\partial^{2} T}{\partial z^{2}}\right)
$$

In above equations, $u, v, w$ are velocities in $x, y, z$ directions, respectively; $t$ is time; $\mu$ is viscosity of air; $P$ is air pressure; $T$ is air temperature; $\rho$ is air density; $c$ is air specific heat; $g$ is acceleration of gravity. Energy equation in the wood block and cylindrical cavity wall is as follows:

$$
\frac{\partial T}{\partial t}=\frac{\lambda}{\rho c}\left(\frac{\partial^{2} T}{\partial x^{2}}+\frac{\partial^{2} T}{\partial y^{2}}+\frac{\partial^{2} T}{\partial z^{2}}\right)
$$

\section{FUZZY LS-SVM}

Support vector machines based on fuzzy least square method (LS-SVMR) was proposed for the purpose of overcoming the defects of large computing amount and poor real-time. It replaced the insensitive function $(\varepsilon)$ with the minimum variance loss function, and also the quadratic programming of SVM could be translated into solving the linear KKT (Karush-Kuhn-Tucker) conditions, so as to simplify the computation. However, the global optimality of the solution under KKT constraints was difficult to guarantee. In order to overcome the non-optimal problem in the whole situation and the over-fitting phenomenon, fuzzy concept is introduced into the paper.

\section{RESUltS AND DisCUSSION}

In this section, the data derived from CFD are picked for training and testing SVM. Although the studied model is 3-D, only temperature field at one 2-D cross-section is studied. In this study, temperature field of the cross-section at $y=0$ is investigated. The heat exchange in the studied enclosure is an unsteady process, i.e. the temperature field in it is changing with time. To simplify the analysis, only temperature field at the 9000s is analyzed (the moment of 9000 s after the heat exchange process begins).

In the SVM model, coordinates $\mathrm{x}$ and $\mathrm{z}$, initial temperature $\mathrm{T}_{0}$, are defined as inputs; while temperature field at 9000s is defined as output. Initial temperatures $\left(\mathrm{T}_{0}\right)$ between $50-130^{\circ} \mathrm{C}$ are studied, in which, Data of $\mathrm{T}_{0}=50^{\circ} \mathrm{C} / 60^{\circ} \mathrm{C} / 70^{\circ} \mathrm{C} / 80^{\circ} \mathrm{C}$ $190^{\circ} \mathrm{C} / 100^{\circ} \mathrm{C} / 110^{\circ} \mathrm{C} / 120^{\circ} \mathrm{C} / 130^{\circ} \mathrm{C}$ are used for training the SVM model, while the data of $\mathrm{T}_{0}=55^{\circ} \mathrm{C} / 65^{\circ} \mathrm{C} / 75^{\circ} \mathrm{C} / 85^{\circ} \mathrm{C}$ $/ 95^{\circ} \mathrm{C} / 105^{\circ} \mathrm{C} / 115^{\circ} \mathrm{C} / 125^{\circ} \mathrm{C}$ are used for testing the model. 


\section{A. Normalization and Error Computation}

In order to improve the generalization ability of LS-SVM, it is necessary to normalize the sample data into $[-1,1]$ or $[0,1]$ to ensure that all variables lie in the same scale, so as to eliminate the magnitude differences between different dimensional data, avoiding big error induced by that. The normalization formulation of coordinate $(x, z)$ and temperature $T$ are as follows:

For coordinate $x$ or $z$ :

$$
\overline{x_{i}}=\frac{x_{i}-x_{\text {mid }}}{\left(x_{\max }-x_{\min }\right) / 2} \text {, in which, } x_{\text {mid }}=\frac{x_{\max }+x_{\min }}{2} \text { (7) }
$$

For temperature $T$ :

$$
\overline{T_{i}}=\frac{T_{i}-T_{\min }}{\left(T_{\max }-T_{\min }\right)}
$$

A model parameter optimization process has been conducted to determine the optimum parameters based on the following performance metrics: mean relative error $(M R E)$, max relative error $(M A E)$, mean square error $(M S E)$ and absolute fraction of variance $\left(R^{2}\right)$. The above errors could be excellent criteria for evaluating the performance of the SVM model. A perfect fit could result in an $\mathrm{R}^{2}$ value of 1 or a very good fit near 1 . The quality of fit decreases as $R^{2}$ decreases.

$$
\begin{gathered}
M R E=\frac{1}{n} \sum_{i=1}^{n} \frac{\left|T_{S V M}-T_{C F D}\right|}{\left(T_{C F D-\max }-T_{C F D-\min }\right)} \\
M A E=\operatorname{Max}\left(\frac{\left|T_{S V M}-T_{C F D}\right|}{T_{C F D-\max }-T_{C F D-\min }}\right) \\
M S E=\sqrt{\frac{1}{n} \sum_{i=1}^{n}\left(T_{S V M}-T_{C F D}\right)^{2} /\left(T_{C F D-\max }-T_{C F D-\min }\right)} \\
R^{2}=1-\frac{\sum_{i=1}^{n}\left(T_{S V M}-T_{C F D}\right)^{2}}{\sum_{i=1}^{n}\left(T_{S V M}-T_{S V M-\text { mean }}\right)^{2}}
\end{gathered}
$$

\section{B. Prediction results}

There are two adjustable parameters in Fuzzy LS-SVM model that correlating with RBF kernel function, i,e. the kernel function parameter $(\sigma)$ and the regularization parameter $(\gamma)$. The two parameters have significant effect on the performance of the SVM model. The higher $\sigma$ could accelerate the converging speed and also improve the generalization ability of the model. The higher $\gamma$ will reduce the smoothness and the generalization ability of the model. In contrast, the lower $\gamma$ could improve the generalization ability, but it also reduce the fitting ability of the model. Therefore, it is necessary to find the optimum values of the two parameters for the training model. Basing on the errors and $\mathrm{R}^{2}$ formulated in Eq.(9)-(12), $\left[1.0 \times 10^{10}, 0.06\right]$ is found to be the optimum parameters of $[\sigma, \gamma]$ by means of cut-and-trial.

In order to make a comparison, another prediction based on BP-ANN method is conducted with the same training data. The well trained BP-ANN model is consisted of three hidden layers, and the number of neurons in each layers are $(15,15,1)$, respectively. The activation function is 'Tan-Sigmoid' and the final performance of the model is 3.62e-06. With the welltrained model, the temperature fields of different $T_{0}$ are predicted. The comparison among the temperature fields of CFD simulation, Fuzzy LS-SVM and BP-ANN predictions under the condition of $\mathrm{T}_{0}=55^{\circ} \mathrm{C}$ are shown in Figure 2 .
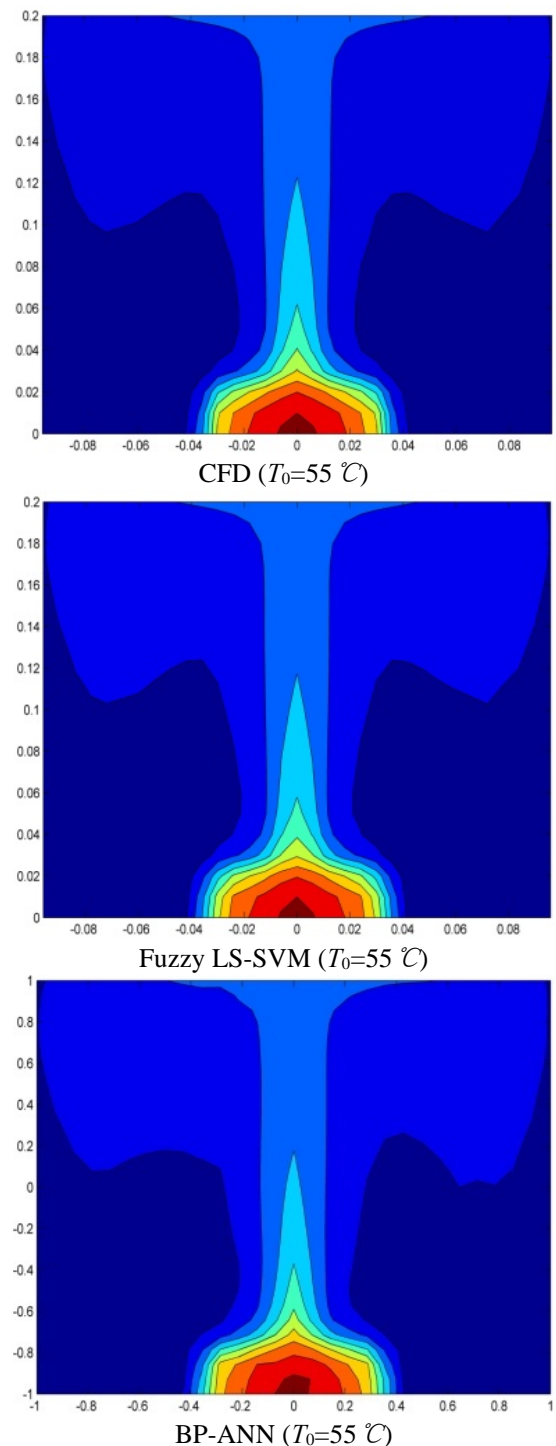

FIGURE II. TEMPERATURE FIELDS COMPARISONS AMONG CFD, FUZZY LS-SVM AND BP-ANN UNDER DIFFERENT INITIAL TEMPERATURES $\left(\mathrm{T} 0=55^{\circ} \mathrm{C}\right)$ 
It can be seen from Figure.2 that both BP-ANN and Fuzzy LS-SVM could effectively predict out the temperature field. Although there are several inconsistent temperature spots existing in the predicted temperature fields, the temperature profiles of the predictions are almost coincide with that of the CFD simulations. The differences between the model predictions and the CFD simulations can be further illustrated with the computed errors such as MRE, MAE, MSE and $R^{2}$. The computed errors and $\mathrm{R}^{2}$ are shown in Table 2 . In order to compare the computation efficiency of the two prediction methods, the time cost of the predictions are also shown in the table.

Table 2 shows that the Fuzzy LS-SVM model is superior to the BP-ANN model in both prediction accuracy and computation efficiency. For example, most MREs of Fuzzy LSSVM is less than that of BP-ANN by an order of magnitude, and the same difference exists in the time cost comparisons between the two models. The reason for this is that BP-ANN is based on the principle of empirical risk minimization, which is easy to fall into local optimum in the classification process of the finite nonlinear data. Besides, there is conflict between the training ability and the prediction ability of BP-ANN, so that the overfitting phenomenon following with small training error and large prediction error is prone to emerge. In contrast, the Fuzzy LS-SVM is based on the VC (Vapnik-Chervonenkis Dimension) theory and the structural risk minimization principle, which could reduce the confidence range of the finite nonlinear data, and also the expectation risk would be reduced in the further step. In another aspect, as analyzed in section 3, it is a linear function rather than a constrained convex quadratic programming to be solved in the Fuzzy LS-SVM model, which could accelerate the computation speed of Fuzzy LS-SVM. To the contrary, the gradient descent algorithm adopted in the BPANN model is prone to slow down the computation speed.

\section{TABLE II. COMPUTED ERRORS AND R2 OF TEMPERATURE FIELD AT CROSS-SECTION OF Y $=0$}

\begin{tabular}{|c|c|c|c|c|c|c|}
\hline $\begin{array}{c}\text { Prediction } \\
\text { method }\end{array}$ & $\begin{array}{c}T_{0} \\
\left({ }^{\circ} \mathrm{C}\right)\end{array}$ & $\begin{array}{c}\text { MRE } \\
(\%)\end{array}$ & $\begin{array}{c}\text { MAE } \\
(\%)\end{array}$ & $\begin{array}{c}\text { MSE } \\
(\%)\end{array}$ & $R^{2}$ & $\begin{array}{c}\text { Time } \\
\text { cost (s) }\end{array}$ \\
\hline \multirow{8}{*}{$\begin{array}{c}\text { Fuzzy LS- } \\
\text { SVM }\end{array}$} & 55 & 0.10 & 0.84 & 0.13 & 0.9983 & 12.80 \\
\hline & 65 & 0.054 & 0.86 & 0.12 & 0.9989 & 12.82 \\
\hline & 75 & 0.058 & 1.02 & 0.14 & 0.9988 & 12.85 \\
\hline & 85 & 0.065 & 1.10 & 0.16 & 0.9987 & 12.72 \\
\hline & 95 & 0.070 & 1.24 & 0.17 & 0.9986 & 12.73 \\
\hline & 105 & 0.082 . & 1.30 & 0.19 & 0.9985 & 12.97 \\
\hline & 115 & 0.15 & 2.60 & 0.27 & 0.9974 & 12.93 \\
\hline & 125 & 0.25 & 1.60 & 0.31 & 0.9968 & 12.77 \\
\hline \multirow{8}{*}{ BP-ANN } & 55 & 0.47 & 3.63 & 0.63 & 0.9983 & 107.06 \\
\hline & 65 & 0.26 & 2.67 & 0.37 & 0.9995 & 107.15 \\
\hline & 75 & 0.23 & 2.28 & 0.34 & 0.9996 & 106.54 \\
\hline & 85 & 0.21 & 2.08 & 0.33 & 0.9997 & 106.63 \\
\hline & 95 & 0.21 & 2.41 & 0.33 & 0.9997 & 106.67 \\
\hline & 105 & 0.22 & 2.55 & 0.34 & 0.9993 & 106.48 \\
\hline & 115 & 0.25 & 5.43 & 0.47 & 0.9996 & 106.46 \\
\hline & 125 & 0.26 & 2.87 & 0.39 & 0.9995 & 106.23 \\
\hline
\end{tabular}

\section{CONCLUSIONS}

The paper conducted a feasibility study of Fuzzy LS-SVM application in the prediction of temperature field induced by natural convection in a cylindrical enclosure. With the simulation data from CFD software, the Fuzzy LS-SVM model is constructed and well trained, and then the untrained CFD data are used for the validation of the prediction results of the model. In order to compare different prediction methods, BPANN is also adopted for the prediction. The validation and comparison are conducted from the mean relative error $(M R E)$, max relative error $(M A E)$, mean square error $(M S E)$ and absolute fraction of variance $\left(R^{2}\right)$. Besides, the prediction time costs of the two prediction methods are also compared with each other. The validation shows that the two prediction methods can both predict out the temperature field effectively. However, the comparison demonstrates that Fuzzy LS-SVM is superior to BP-ANN in both prediction accuracy and computation efficiency.

\section{ACKNOWLEDGMENT}

This work is financially sponsored by Taishan Scholar Project of Shandong Province of China.

\section{REFERENCES}

[1] M. Ge, R. Du, G.C. Zhang, Y. Xu. "Fault diagnosis using support vector machine with an application in sheet metal stamping operations," Mechanical Systems \& Signal Processing. 18 (2004), pp.143-159.

[2] S. R. Sain, The Nature of Statistical Learning Theory, Springer 1995, 8 (6), pp.988 -999.

[3] A. Paniagua-Tineo, S. Salcedo-Sanz, C. Casanova-Mateo, et al. "Prediction of daily maximum temperature using a support vector regression algorithm," Renewable Energy 36 (2011), pp.3054-3060.

[4] Q. Cai, W.Wang, S. Wang. “Application of Indoor Temperature Prediction Based on SVM and BPNN," Control \& Decision Conference (2015), pp. 2883-2887

[5] M.A. Mohandes, T.O. Halawani, S. Rehman, A.A.Hussain. "Support vector machines for wind speed prediction," Renewable Energy 29 (2004) , pp.939-947.

[6] L. Chen, H.B. Liu, W. Wu, D. Xie. "Estimation of monthly solar radiation from measured temperatures using support vector machines - A case study,” Renewable Energy 36(2011), pp.413-420.

[7] Y.Varol, H.F. Oztop, E.Avci. "Estimation of thermal and flow fields due to natural convection using support vector machines (SVM) in a porous cavity with discrete heat sources," International Communications in Heat and Mass Transfer 35 (2008), pp.928-936.

[8] Y. Varol, H.F. Oztop, A. Koca, E. Avci. "Forecasting of entropy production due to buoyant convection using support vector machines (SVM) in a partially cooled square cross-sectional room," Expert Systems with Applications 36 (2009), pp.5813-5821.

[9] [9] A. Zendehboudi, B. Wang, X. Li. “Application of smart models for prediction of the frost layer thickness on vertical cryogenic surfaces under natural convection,” Applied Thermal Engineering 115 (2017), pp.1128-1136.

[10] J. L. Lage, A. Bejan. "The Ra-Pr domain of laminar natural convection in an enclosure heated from the side," Numerical Heat Transfer Applications. Part A 19 (1991), pp. 21-41.

[11] E. Ntibarufata, M. Hasnaoui, E. Bilgen, P Vasseur. "Natural convection in partitioned enclosures with localized heating," International Journal of Numerical Methods for Heat \& Fluid Flow 3 (1993), pp. 133-143.

[12] D. Das, M. Roy, T. Basak. "Studies on natural convection within enclosures of various (non-square) shapes - A review," International Journal of Heat and Mass Transfer 106 (2017), pp.356-406.

[13] B. Calcagni, F. Marsili, M. Paroncini. "Natural convective heat transfer in square enclosures heated from below," Applied Thermal Engineering 25 (2005), pp. 2522-2531. 